AdVANCES IN COMBINATORICS, 2020:11, 12 pp.

www.advancesincombinatorics.com

\title{
Unitary Signings and Induced Subgraphs of Cayley Graphs of $\mathbb{Z}_{2}^{n}$
}

\author{
Noga Alon* Kai Zheng \\ Received 26 March 2020; Published 9 November 2020
}

\begin{abstract}
Let $G$ be the Cayley graph of the elementary abelian 2-group $\mathbb{Z}_{2}^{n}$ with respect to a set $S$ of size $d$. We prove that for any such $G, S$ and $d$, the maximum degree of any induced subgraph of $G$ on any set of more than half the vertices is at least $\sqrt{d}$. This is deduced from the recent breakthrough result of Huang who proved the above for the $n$-hypercube $Q^{n}$, in which the set of generators $S$ is the set of all vectors of Hamming weight 1. Motivated by his method we define and study unitary signings of adjacency matrices of graphs, and compare them to the orthogonal signings of Huang. As a byproduct, we answer a recent question of Belardo, Cioabă, Koolen, and Wang about the spectrum of signed 5-regular graphs.
\end{abstract}

\section{Introduction}

For a group $B$ and a set $S \subseteq B$ satisfying $S=S^{-1}$, the Cayley graph of $B$ with a generating set $S$, denoted $\Gamma(B, S)$, is the graph whose set of vertices is the set of elements of $B$, in which the set of edges is the set of all (unordered) pairs $\{g, g s\}$ where $g \in B$ and $s \in S$. Any Cayley graph is regular and vertex transitive. In this note we consider Cayley graphs of the elementary abelian 2-group $B=\mathbb{Z}_{2}^{n}$. Here we use additive notation and note that $S=-S$ for every subset of the group. A particular example that has been the subject of recent attention is the $n$-dimensional hypercube, $Q^{n}$. This is the Cayley graph of $B=\mathbb{Z}_{2}^{n}$ whose generating set $S$ consists of all length $n$ boolean vectors with Hamming weight 1 . A recent breakthrough result of Huang [9] asserts that any induced subgraph $H$ of $Q^{n}$ on a set of $2^{n-1}+1$ vertices has maximum degree $\Delta(H)$ at least $\sqrt{n}$. This, together with a result of Gotsman and Linial [7], confirmed the Sensitivity Conjecture of Nisan and Szegedy [14].

\footnotetext{
* Supported in part by NSF grant DMS-1855464, ISF grant 281/17, BSF grant 2018267 and the Simons Foundation.
} 
Huang's result also improves a previous lower bound of $\left(\frac{1}{2}-o(1)\right) \log _{2} n$ of Chung, Füredi, Graham, and Seymour [6], while another result of [6] shows that his $\sqrt{n}$ bound is tight.

Our first result extends the lower bound to any Cayley graph of $\mathbb{Z}_{2}^{n}$. These graphs are sometimes called cubelike graphs, see, for example, [13].

Theorem 1.1. For any Cayley graph $G=\Gamma\left(\mathbb{Z}_{2}^{n}, S\right)$ of $\mathbb{Z}_{2}^{n}$ with respect to any generating set $S$, and for any subset $U \subseteq \mathbb{Z}_{2}^{n}$ of cardinality $|U|>2^{n-1}$, the maximum degree of the induced subgraph $H$ of $G$ on $U$ satisfies $\Delta(H) \geq \sqrt{|S|}$.

The proof, presented in the next section, is a simple application of Huang's result.

To prove his result, Huang shows that $Q^{n}$ admits an orthogonal signing. This is a matrix obtained from the adjacency matrix of $Q^{n}$ by replacing every nonzero entry by 1 or -1 so that the resulting matrix, call it $A$, is symmetric and has orthogonal rows. Therefore, each eigenvalue of this matrix is either $+\sqrt{n}$ or $-\sqrt{n}$, and as the trace is zero, exactly half the eigenvalues are $+\sqrt{n}$ and half are $-\sqrt{n}$. By Cauchy's Interlace Theorem (see, e.g., [10] for a short proof) this implies that the largest eigenvalue of any principal minor of size at least $2^{n-1}+1$ of $A$ is $\sqrt{n}$. The result about the maximum degree of any induced subgraph of $Q^{n}$ on a set $U$ of more than half the vertices follows from the simple fact that any eigenvalue of a matrix in which all nonzero entries have absolute value 1 is at most the size of the largest support of a row of the matrix.

Motivated by this proof we define a unitary signing ${ }^{1}$ of a graph. This is a Hermitian matrix obtained from the adjacency matrix of the graph by replacing each nonzero entry by a complex number of norm 1 , so that the (complex) inner product of any two distinct rows is 0 . Huang's argument works in the complex case as well, implying that if a $d$-regular graph admits a unitary signing, then the maximum degree of any induced subgraph of it on a set of more than half the vertices is at least $\sqrt{d}$. Unitary signings of graphs appear to be interesting in their own right.

In Section 3 we prove the following result, providing additional examples of Cayley graphs of $\mathbb{Z}_{2}^{n}$ with orthogonal and unitary signings.

Theorem 1.2. Let $T=\left\{e_{1}, \ldots, e_{n}\right\}$ and let $U=\left\{E_{2}, \ldots, E_{n}\right\}$ where $e_{i}$ is the length $n$ boolean vector of Hamming weight 1 with 1 in its ith coordinate and $E_{i}=\sum_{k=1}^{k=i} e_{k}$. Let $G$ be a Cayley graph of $\mathbb{Z}_{2}^{n}$ with a generating set $S \subseteq T \cup U$ so that $|S \cap U| \leq 1$. Then $G$ admits a unitary signing.

Regarding the necessity of using complex numbers in unitary signings, we show in Section 4 that some graphs have unitary signings but do not have orthogonal signings. Let $Q_{+}^{n}$ denote the Cayley graph of $\mathbb{Z}_{2}^{n}$ with the generating set $S=\left\{e_{1}, \ldots, e_{n}, E_{n}\right\}$.

Proposition 1.3. For $n \geq 2, Q_{+}^{n}$ has an orthogonal signing if and only if $n \equiv 0,3(\bmod 4)$. In particular for $n \equiv 1,2(\bmod 4), Q_{+}^{n}$ has a unitary signing but no orthogonal signing.

Finally, as a byproduct we answer a question of Belardo, Cioabă, Koolen, and Wang [4] about the spectrum of signed 5-regular graphs.

\footnotetext{
${ }^{1}$ While similar terminology is used, orthogonal and unitary signings are not in general orthogonal or unitary matrices, which are matrices $M$ such that $M M^{t}$ or $M M^{*}$ is the identity.
} 
Unitary Signings and InduCED Subgraphs of CAYLEy GRAPHS of $\mathbb{Z}_{2}^{n}$

\section{The proof of Theorem 1.1}

Proof of Theorem 1.1: Let $G$ be a Cayley graph of $\mathbb{Z}_{2}^{n}$ with generating set $S=\left\{s_{1}, s_{2}, \ldots, s_{d}\right\}$. We may and will assume without loss of generality that $G$ is connected, that is, the vectors of $S$ span the vector space $\mathbb{Z}_{2}^{n}$. Indeed, otherwise the graph $G$ consists of isomorphic connected components on the cosets of $\operatorname{Span}(S)$. Any set of more than half the vertices of $G$ contains more than half the vertices of at least one of these components, and we can thus deduce the result from the one for the connected case.

Let $Q^{d}$ be the $d$-dimensional hypercube. This is the Cayley graph of $\mathbb{Z}_{2}^{d}$ with respect to the set of generators $\left\{e_{1}, e_{2}, \ldots, e_{d}\right\}$ consisting of all the vectors of Hamming weight 1 in $\mathbb{Z}_{2}^{d}$. Let $T$ be the linear transformation from $\mathbb{Z}_{2}^{d}$ to $\mathbb{Z}_{2}^{n}$ defined by $T\left(e_{i}\right)=s_{i}$ for all $1 \leq i \leq d$. Since $S$ spans $\mathbb{Z}_{2}^{n}, T$ is onto, and the inverse image of any element of $\mathbb{Z}_{2}^{n}$ contains exactly $2^{d-n}$ elements. Let $U \subseteq \mathbb{Z}_{2}^{n}$ be a set of more than half the vertices of $G$. Then the inverse image $W=T^{-1}(U)$ of $U$ contains more than half the elements of $\mathbb{Z}_{2}^{d}$. By Huang's result there are distinct $w, w_{1}, w_{2}, \ldots, w_{t} \in W$, with $t \geq \sqrt{d}$, such that $w$ is adjacent in $Q^{d}$ to each $w_{i}, 1 \leq i \leq t$. Therefore, the vertex $T(w) \in U$ is adjacent in $G$ to each of the $t \geq \sqrt{d}$ distinct vertices $T\left(w_{1}\right), T\left(w_{2}\right), \ldots, T\left(w_{t}\right)$, which all lie in $U$. This completes the proof.

\section{Unitary signings of graphs}

As mentioned in the introduction, we consider here unitary and orthogonal signings of graphs, defined as follows.

Definition 3.1. A unitary signing of a graph is a Hermitian matrix obtained from the adjacency matrix of the graph by replacing each nonzero entry by a complex number of norm 1, so that the (complex) inner product of any two distinct rows is 0 . If all nonzero entries used are real, that is, all are +1 or -1 , this is an orthogonal signing.

It is shown in [9] that the hypercube $Q^{n}$ admits an orthogonal signing. We extend this result in Theorem 1.2 which is proved in this section. Note that any Cayley graph of $\mathbb{Z}_{2}^{n}$ with a generating set consisting of $n+1$ elements which span $\mathbb{Z}_{2}^{n}$ is isomorphic to one of the graphs described in this theorem.

A useful fact about unitary signings of Cayley graphs of $\mathbb{Z}_{2}^{n}$ is that we can view them as sums of what may be called edge signings, as stated in the next simple lemma.

Lemma 3.1. Let $G=\Gamma\left(\mathbb{Z}_{2}^{n}, S\right)$, and suppose $S=\left\{a_{1}, \ldots, a_{m}\right\}$ where each $a_{j}$ is distinct. If $A_{j}$ is a unitary signing of $\Gamma\left(\mathbb{Z}_{2}^{n},\left\{a_{j}\right\}\right)$ and the matrices $A_{j}$ anticommute, then $A=\sum_{j=1}^{j=m} A_{j}$ is a unitary signing of $G$.

Proof. Obvious from the definition.

We refer to a signing of a single element Cayley graph, $\Gamma\left(\mathbb{Z}_{2}^{n},\{e\}\right)$, as simply a signing of the edge $e$ or an edge signing of $e$. If the signing is also a unitary signing, we may call it a unitary signing of $e$ or a unitary edge signing. Such a Cayley graph is a perfect matching on $\mathbb{Z}_{2}^{n}$.

A convenient way of constructing edge signings when $n \geq 2$ is to use Kronecker products of the following $2 \times 2$ Hermitian matrices. The first one is the identity, and the other three are known as Pauli 
matrices, and generate the Clifford Algebra of $\mathbb{R}^{3}$.

$$
\begin{aligned}
I_{2} & =\left[\begin{array}{ll}
1 & 0 \\
0 & 1
\end{array}\right], \\
R_{0} & =\left[\begin{array}{cc}
1 & 0 \\
0 & -1
\end{array}\right], \\
R_{1} & =\left[\begin{array}{ll}
0 & 1 \\
1 & 0
\end{array}\right], \\
R_{2} & =\left[\begin{array}{cc}
0 & i \\
-i & 0
\end{array}\right] .
\end{aligned}
$$

With this notation, it is clear that $A_{n} \otimes \cdots \otimes A_{1}$ is a unitary signing of $e=\left(a_{n}, \ldots, a_{1}\right)$ if $A_{j} \in\left\{I_{2}, R_{0}\right\}$ whenever $a_{j}=0$, and $A_{j} \in\left\{R_{1}, R_{2}\right\}$ whenever $a_{j}=1$. We will sometimes refer to $A_{i}$ as the matrix in the $i$ th position of the Kronecker product. Moreover, we will also make liberal use of the fact that $\left\{R_{0}, R_{1}, R_{2}\right\}$ is an anticommuting family while all other pairs of the above four matrices commute. When combined with the mixed product property, this fact provides an easy way to check if two signings of the above form anticommute. Indeed, if $A=A_{n} \otimes \cdots \otimes A_{1}$ and $B=B_{n} \otimes \cdots \otimes B_{1}$, with all $A_{i}, B_{i} \in\left\{I_{2}, R_{0}, R_{1}, R_{2}\right\}$, then $A$ and $B$ either anticommute or commute. They anticommute if and only if an odd number of the pairs $A_{i}, B_{i}$ for $1 \leq i \leq n$ anticommute. In order to prove Theorem 1.2 we construct anticommuting families of unitary edge signings using this method.

Lemma 3.2. For any generating set $S \subseteq \mathbb{Z}_{2}^{n}$ as described in Theorem 1.2, there exists an anticommuting family of unitary edge signings $\left\{A_{e}\right\}_{e \in S}$.

Proof. Let $A_{e_{i}}=M_{n} \otimes \cdots \otimes M_{1}$ where

$$
\begin{cases}M_{j}=R_{0}, & \text { if } \quad j>i \\ M_{j}=R_{1}, & \text { if } \quad j=i \\ M_{j}=I_{2}, & \text { if } \quad j<i .\end{cases}
$$

It is not difficult to check that $\left\{A_{e_{i}}\right\}_{i \in[n]}$ is an anticommuting family of unitary edge signings where $[n]$ denotes the integers from 1 to $n$. In fact, they are also orthogonal signings and $\sum_{i=1}^{i=n} A_{e_{i}}$ is the matrix that Huang constructs in Lemma 2.2 of [9]. To finish the proof, it remains to show that for any $E_{i} \in U$, there is a unitary signing for the edge $E_{i}$ that anticommutes with each matrix in $\left\{A_{e_{i}}\right\}_{i \in[n]}$. First define $B_{k}=M_{k} \otimes \cdots \otimes M_{1}$ where

$$
\begin{cases}M_{j}=R_{1}, & \text { if } k-j \text { is odd } \\ M_{j}=R_{2}, & \text { if } k-j \text { is even. }\end{cases}
$$

The key property of the expansion of $B_{k}$ is that each $R_{1}$ has an odd number of matrices to the left of it, while each $R_{2}$ has an even number of matrices to the left of it. $E_{i}$ 's edge signing is as follows,

$$
A_{E_{i}}=\underbrace{R_{0} \otimes \cdots \otimes R_{0}}_{n-i \text { times }} \otimes B_{i}
$$




\section{UNITARY SigNiNGS AND INDUCED SUBGRAPHS OF CAYLEY GRAPHS OF $\mathbb{Z}_{2}^{n}$}

To check that any $\left\{A_{E_{i}}\right\} \cup\left\{A_{e_{i}}\right\}_{i \in[n]}$ is an anticommuting family of size $n+1$ we show that $A_{E_{i}}$ and $A_{e_{j}}$ anticommute for any $i, j$. We count the number of anticommuting pairs of matrices in their Kronecker product expansions. If $i \leq j$ there is one anticommuting pair: the $R_{1}$ from $A_{e_{j}}$ with either an $R_{0}$ if $i<j$ or an $R_{2}$ if $i=j$.

If $i>j$ then $A_{e_{j}}$ has $R_{1}$ in the $j$ th position, while $A_{E_{j}}$ can have either $R_{1}$ or $R_{2}$. If it has $R_{1}$ as well, then the $j$ th position is not an anticommuting pair, but we have an odd number of $R_{2}$ and $R_{1}$ 's to the left of the $j$ th position as mentioned above. These $R_{2}$ and $R_{1}$ matrices all anticommute with the corresponding $R_{0}$ 's in $A_{e_{i}}$ 's expansion. Likewise, if we have $R_{2}$ in the $j$ th position, then all the $R_{1}$ 's and $R_{2}$ 's in $B_{i}$ to the left of and including the $j$ th position form anticommuting pairs and again this is an odd number of pairs. In each case every other pair commutes as it is either $R_{0} R_{0}$ or a pair with one of the matrices being $I_{2}$.

We can now prove Theorem 1.2. Let $G$ be a Cayley graph of $\mathbb{Z}_{2}^{n}$ with generating set $S$ as described in the theorem. By Lemma 3.2, there is a family of anticommuting matrices consisting of unitary signings for the edges in $S$. The sum of these matrices is a unitary signing of $G$ by Lemma 3.1.

It is worth noting that there are additional pairs of anticommuting matrices outside of the family described in Lemma 3.2. This leads to unitary signings of certain Cayley graphs of $\mathbb{Z}_{2}^{n}$ with generating sets consisting of vectors from $T \cup U$ and up to two vectors from $U$.

Corollary 3.3. Any Cayley graph of $\mathbb{Z}_{2}^{n}$ with generating set consisting of a subset of $T=\left\{e_{1}, \ldots, e_{n}\right\}$ and exactly two vectors $E_{i}, E_{j} \in U=\left\{E_{2}, \ldots, E_{n}\right\}$ such that $i>j, i$ is odd and $j$ is even, admits a unitary signing.

Proof. Using the construction from Lemma 3.2, it is easy to check that if $i>j, i$ is odd and $j$ is even then $A_{E_{i}}$ and $A_{E_{j}}$ anticommute. Thus, the edge signings constructed in Lemma 3.2 provide the desired unitary signing.

\section{Unitary versus orthogonal signings}

One may wonder if the use of complex numbers is needed in the proof of Theorem 1.2. We show that complex signings are indeed necessary in some cases. Recall that $Q_{+}^{n}=\Gamma\left(\mathbb{Z}_{2}^{n}, S\right)$ is the Cayley graph of $\mathbb{Z}_{2}^{n}$ with $S=\left\{e_{1}, \ldots, e_{n}, E_{n}\right\}$. This graph is simply the $n$-dimensional hypercube with one additional element being the all 1 vector added to its generating set. In this section we start with the proof of Proposition 1.3 stated in Section 1.

The reverse direction of this Proposition is immediate as for $n \equiv 0,3(\bmod 4)$ and $n \geq 2$, the unitary signing of $Q_{+}^{n}$ constructed in Theorem 1.2 only uses real numbers and is thus an orthogonal signing as well.

To complete the proof of Proposition 1.3 we first discuss a simple way to determine whether certain graphs have an orthogonal signing. The method works whenever the graph in question has the property that any two vertices with a common neighbor have exactly two common neighbors. In Cayley graphs of $\mathbb{Z}_{2}^{n}$ this holds if and only if the generating set of the graph is a Sidon set. A Sidon set is a set with the property that any distinct pair of elements has a unique sum, that is $s_{1}+s_{2}=s_{3}+s_{4}$ if and only if $\left\{s_{1}, s_{2}\right\}=\left\{s_{3}, s_{4}\right\}$ for all $s_{1}, s_{2}, s_{3}, s_{4} \in S$ satisfying $s_{1} \neq s_{2}$ and $s_{3} \neq s_{4}$. Note that the generating set of $Q^{n}$ is a Sidon set and the generating set of $Q_{+}^{n}$ is a Sidon set when $n \neq 3$. Thus, the graph $Q_{+}^{n}$ with 
$n \geq 2$ and $n \equiv 1,2(\bmod 4)$ has the property that vertices with a common neighbor have exactly two common neighbors. It is worth noting that there are other well known strongly-regular or distance-regular graphs with this property, including the Shrikhande graph, the 2-dimensional Hamming graph over $\mathbb{Z}_{4}$, the Gewirtz graph, and the Klein graph, see [5].

Given an orthogonal signing of the adjacency matrix $M$ of a graph, one can define a labelling, $f$, of the edges of the graph using 1's and 0's as follows. Let $e=i j$ be an edge, then put

$$
f(e)= \begin{cases}1, & \text { if } M_{i j}=-1 \\ 0, & \text { otherwise }\end{cases}
$$

For Cayley graphs $\Gamma\left(\mathbb{Z}_{2}^{n}, S\right)$ where $S$ is a Sidon set, we can then reformulate the problem of finding orthogonal signings as follows.

Lemma 4.1. Let $G=\Gamma(B, S)$ be a Cayley graph of $B=\mathbb{Z}_{2}^{n}$ where $S$ is a Sidon set. Let $C$ be the set of all 4 -cycles in $G$ where the elements of $C$ are sets of 4 edges that form a 4 cycle. An orthogonal signing of $G$ exists if and only if there exists a labelling of the edges of the graph $f: E(G) \rightarrow\{0,1\}$, such that $\sum_{e \in c} f(e)=1$ for all cycles $c \in C$, where each sum here is computed in the group $\mathbb{Z}_{2}$.

Proof. Since $S$ is a Sidon set, $G$ has the property that any two vertices with a common neighbor share exactly two common neighbors. Let $M_{i j}$ be the entry of the edge $e=i j$. If an orthogonal signing exists, then for any 4-cycle, in order for the rows indexed by opposite vertices of the 4-cycle to be orthogonal, the 4-cycle must have exactly 3 edges whose entry is 1 or exactly 3 edges whose entry is -1 . The desired labelling of $f$ is thus the one described above.

On the other hand, if such a labelling $f$ exists, one can define the signed adjacency matrix $M$ by $M_{i j}=-1$ if the edge $i j$ is labelled 1 and $M_{i j}=1$ if the edge $i j$ is labelled 0 . Then the inner products of rows $i$ and $j$ is the sum, over all length 2 walks between $i$ and $j$, of the product of the edge entries of each walk. There are either 2 or 0 length 2 walks between any two vertices. The result then follows from the assumption that in each 4-cycle there is an odd number of edges labelled 1.

We can now prove the remaining direction in Proposition 1.3.

Proof. To show that $Q_{+}^{n}$ has no orthogonal signing when $n \geq 2$ and $n \equiv 1,2(\bmod 4)$, it suffices, by Lemma 4.1, to find an odd number of cycles such that each edge used in a cycle is used in an even number of cycles. Indeed, let $C_{1}, \ldots, C_{2 k+1}$ be such a family cycles. If an orthogonal signing exists, we have $\sum_{e \in C_{i}} f(e)=1$, for each $C_{i}$. Adding the $2 k+1$ equations together yields $\sum_{e \in \cup_{i=1}^{i=2 k+1} C_{i}} f(e)=1$. However, since each edge is used an even number of times, the left hand side is 0 , yielding a contradiction.

Thus, it remains to show that an odd collection of cycles as above exists. We describe such a collection arranged in a staircase shape. This collection in $Q_{+}^{5}$ is depicted in Figure 1 and the general construction, described in what follows, is similar.

Consider the integer lattice with all coordinates $(x, y)$ such that $x+y \leq n+1$ and $n \geq x, y \geq 0$. Notice that connecting adjacent lattice points gives a staircase grid composed of $\frac{n(n+1)}{2}$ unit squares. We can view this structure as a graph $L_{n}$ whose set of vertices is $V\left(L_{n}\right)=\{(x, y): x+y \leq n+1, n \geq x, y \geq 0\}$ where $\left(x_{1}, y_{1}\right)$ is adjacent to $\left(x_{2}, y_{2}\right)$ if and only if the two lattice points are exactly one unit apart. It is easy to see that the 4-cycles of $L_{n}$ correspond exactly to the unit squares of the staircase shape and that as 


\section{Unitary Signings and InduCED Subgraphs of CAYley Graphs of $\mathbb{Z}_{2}^{n}$}

a result $L_{n}$ has $\frac{n(n+1)}{2}$ 4-cycles. We identify each vertex $(x, y) \in V\left(L_{n}\right)$ with a vertex in $V\left(Q_{+}^{n}\right)$ such that the edges of $L_{n}$ are preserved. Define $f: V\left(L_{n}\right) \rightarrow\{0,1\}^{n}$ to be this mapping. Let $S=\left\{e_{1}, \ldots, e_{n}, E_{n}\right\}$ be the generating vectors of $Q_{+}^{n}$ as defined previously. Write $f(x, y)$ instead of $f((x, y))$, for simplicity and use $(a, b)$ to denote the edge between $a$ and $b$ when $a$ and $b$ are vertices (in $L_{n}$ or in $f\left(L_{n}\right)$ ).

Define

$$
\begin{array}{llrl}
f(0,0) & =(0, \ldots, 0), & & \\
f(x, 0) & =e_{1}+\ldots+e_{x}, & & \text { for } x \geq 1 \\
f(0, y) & =e_{1}+\ldots+e_{n+1-y}, & & \text { for } y \geq 1 \\
f(x, y) & =f(x, 0)+f(0, y) . & &
\end{array}
$$

It is clear that $f$ preserves the edges of $L_{n}$, as $f(x+1, y)-f(x, y), f(x, y+1)-f(x, y) \in S$. Moreover if the vertices $\{(x, y),(x+1, y),(x, y+1),(x+1, y+1)\}$ form a 4-cycle in $L_{n}$, then $x+1+y+1 \leq n+1$ and

$$
f(x+1, y)-f(x, y)=e_{x+1} \neq e_{n+1-y}=f(x, y+1)-f(x, y) .
$$

Thus, no 4-cycle of $L_{n}$ is mapped to a degenerate 4-cycle, so $f\left(L_{n}\right)$ yields a collection of $\frac{n(n+1)}{2}$ 4-cycles in the graph $Q_{+}^{n}$. For $n \equiv 1,2(\bmod 4)$ this is an odd number, so it remains to show that this collection of cycles uses each edge an even number of times. We can pair the edges of the 4-cycles of $L_{n}$ so that in each pair, the two edges have the same image under $f$. This implies that the images of the $\frac{n(n+1)}{2} 4$-cycles of $L_{n}$ under $f$ form an odd collection of 4-cycles with each edge used an even number of times in $Q_{+}^{n}$. Note, first, that any edge in the interior can be paired with itself since it is used in two cycles. Next, for the boundary edges note that $((0,0),(1,0))$ can be paired with $((0, n),(1, n))$ as

$$
\begin{aligned}
& f(1, n)=e_{1}+e_{1}=0=f(0,0), \\
& f(1,0)=e_{1}=f(0, n) .
\end{aligned}
$$

Similarly, $((0,0),(0,1))$ can be paired with $((n, 0),(n, 1))$ as

$$
\begin{aligned}
& f(n, 1)=E_{n}+E_{n}=0=f(0,0), \\
& f(n, 0)=E_{n}=f(0,1) .
\end{aligned}
$$

We can also pair the edge $((x, 0),(x+1,0))$ with $((0, n+1-x),(0, n-x))$ for $1 \leq x \leq n-1$ as

$$
\begin{aligned}
f(x, 0) & =e_{1}+\cdots+e_{x}=f(0, n+1-x), \\
f(x+1,0) & =e_{1}+\cdots+e_{x+1}=f(0, n-x) .
\end{aligned}
$$

Lastly it remains to pair the edges of the form $((x, y),(x, y+1))$ and $((x, y),(x+1, y))$ for $1 \leq x, y \leq n-1$ and $x+y+1=n+1$. These are the edges on the boundary, but not on one of the axes. Notice that $((x, y),(x+1, y))$ and $((x, y),(x, y+1))$ are mapped to the same edge if $x+y+1=n+1$. Indeed both are mapped to the edge $\left(e_{x+1}, 0\right)$. This exhausts all edges on the boundary implying that under $f\left(L_{n}\right)$, each 
edge appears in an even number of cycles. Thus, for $n \equiv 1,2(\bmod 4)$, we get a collection of $\frac{n(n+1)}{2} \equiv 1$ ( $\bmod 2)$ cycles where each edge is used an even number of times. By Lemma 4.1, this implies that there is no real orthogonal signing of $Q_{+}^{n}$ when $n \equiv 1,2(\bmod 4)$. However, by Theorem 1.2, a complex unitary signing does exist.

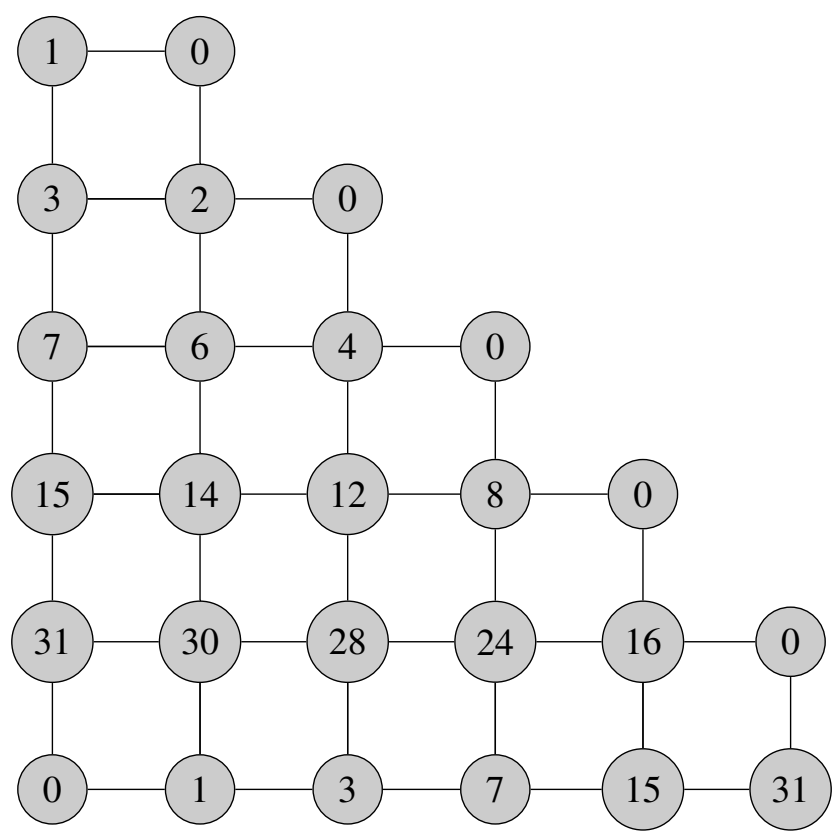

Figure 1: A depiction of $f\left(L_{5}\right)$, or 15 cycles in $Q_{+}^{5}$ where each edge is used two times. The vertex labels correspond to their binary representations as length 5 Boolean vectors, e.g. 15 is the vertex $(0,1,1,1,1)$.

Notice that the construction above works whenever we have a cycle of length 2 or 3 modulo 4 with distinct generators for a Cayley graph of $\mathbb{Z}_{2}^{n}$. We simply repeat the above, using such a cycle of generators in place of $\left\{e_{1}, \ldots, e_{n}, E_{n}\right\}$. Thus, we get the following result on the existence of orthogonal signings for Cayley graphs of $\mathbb{Z}_{2}^{n}$.

Proposition 4.2. Let $G$ be a Cayley graph of $\mathbb{Z}_{2}^{n}$ with generating set $S$. If there exist distinct $e_{1}, \ldots, e_{k} \in S$ such that $e_{1}+\cdots+e_{k}=0$, and $k \equiv 2,3(\bmod 4)$, then no orthogonal signing of $G$ exists.

Proof. Immediate from the construction in the previous proof using the edges $e_{1}, \ldots, e_{k}$.

It is interesting to note that our signing of $Q_{+}^{4}$, which, as can be easily checked, uses only real numbers, answers an open question posed recently by Belardo, Cioabă, Koolen, and Wang [4]. They studied real orthogonal signings of regular graphs and noted that any 4-regular graph with an orthogonal signing, $A_{s}$, can be used to produce a 5-regular graph with an orthogonal signing,

$$
B=\left[\begin{array}{cc}
A_{S} & I \\
I & -A_{S}
\end{array}\right]
$$




\section{UNITARY SigNingS AND INDUCED SUbGRAPHS OF CAYLEY GRAPHS OF $\mathbb{Z}_{2}^{n}$}

in a way similar to Huang's matrix construction. Specifically, the new 5-regular graph is formed by taking two copies of the 4-regular graph and adding a perfect matching between them joining each vertex to its copy. By the work of McKee and Smyth [12] on 4-regular graphs, this method yields many 5-regular graphs with such an orthogonal signed adjacency matrix. Belardo, Cioabă, Koolen, and Wang ask if any other such examples exist, namely if there are 5-regular graphs with orthogonal signings that are not constructed by the above method. Our graph $Q_{+}^{4}$ is one such example. To show it is not one of the examples described in [4] note that all the graphs there have a cut forming a perfect matching which splits the vertex set into two equal parts. For $Q_{+}^{4}$ to be such a graph it would have to have a cut of size 8 . However, this is not the case as can be seen from the eigenvalues of its adjacency matrix. This follows from the following known fact, proved, for example, in the remark following Lemma 2.1 in [2]. For completeness we include the simple proof.

Proposition 4.3. If $G=(V, E)$ is a regular graph with $|V|=n$ even, $U \subseteq V,|U|=\frac{n}{2}$, and $G$ has eigenvalues $\lambda_{1} \geq \cdots \geq \lambda_{n}$, then $e(U, V-U) \geq \frac{d-\lambda_{2}}{4} n$, where $e(U, V-U)$ denotes the number of edges with endpoints in $U$ and $V-U$.

Proof. Let $A$ be the adjacency matrix of $G$ and let $f$ be the column vector indexed by $V$ defined by:

$$
f(u)= \begin{cases}1, & \text { if } u \in U \\ -1, & \text { if } u \notin U,\end{cases}
$$

where $f(u)$ is the coordinate of $f$ indexed by $u \in V$. We have,

$$
f^{t} A f=2 \sum_{u v \in E} f(u) f(v)=2\left[-e(U, V-U)+\frac{n d}{2}-e(U, V-U)\right]=n d-4 e(U, V-U)
$$

and hence

$$
e(U, V-U)=\frac{n d}{4}-\frac{f^{t} A f}{4}
$$

One can bound $f^{t} A f$ using the second largest eigenvalue of $A$ since it is orthogonal to the all 1 vector which is the eigenvector of the largest eigenvalue. Thus, $f^{t} A f \leq \lambda_{2}\|f\|_{2}^{2}=\lambda_{2} n$, and

$$
e(U, V-U) \geq \frac{n d}{4}-\frac{\lambda_{2} n}{4}=\frac{d-\lambda_{2}}{4} n \text {. }
$$

It is well known (c.f., for example, [11], Problem 11.8) that the eigenvalues of Cayley graphs of Abelian groups can be expressed as character sums. This easily implies that the second eigenvalue of $Q_{+}^{4}$ is 1 . Thus, by the previous proposition, any cut of $Q_{+}^{4}$ that splits the vertex set into two equal vertex classes has at least 16 edges. However, a perfect matching of $Q_{+}^{4}$ has exactly 8 edges, so $Q_{+}^{4}$ cannot have the form described in [4] in which there is such a cut. 


\section{Concluding remarks}

By Theorem 1.1 the induced subgraph of any $d$-regular Cayley graph of $\mathbb{Z}_{2}^{n}$ on more than half the vertices has maximum degree at least $\sqrt{d}$. It is easy to see that this is not the case for Cayley graphs of other (abelian) groups. For example, if a group has an element of order 6 then the Cayley graph in which the only generators are such an element and its inverse is a vertex disjoint union of 6-cycles. This has a set of two thirds of the vertices so that the induced subgraph on them is a matching, having maximum degree $1<\sqrt{2}$.

The lower bound provided by Theorem 1.1 is tight for the $n$-cube, as shown by the example described in [6]. It is therefore also tight for any Cayley graph obtained from the $n$ cube by adding one additional generator, when $n$ is a perfect square, and is off by at most one for other $n$. For many Cayley graphs of $\mathbb{Z}_{2}^{n}$, however, the bound is far from being tight. In particular for most Cayley graphs with at least $C n$ generators, for some absolute constant $C$, the lower bound can be improved to linear in $d$. This follows from known results about expanders and random Cayley graphs, as explained in what follows. An $(n, d, \lambda)$-graph is a $d$-regular graph on $n$ vertices in which the absolute value of every eigenvalue besides the top one is at most $\lambda$. The following result is proved in [1].

Lemma 5.1 ([1]). Let $G=(V, E)$ be an $(n, d, \lambda)$ graph and let $S$ be an arbitrary set of $\alpha$ vertices of $G$. Then the average degree $\bar{d}$ of the induced subgraph of $G$ on $S$ satisfies

$$
|\bar{d}-\alpha d| \leq \lambda(1-\alpha)
$$

The proof in [1] in fact shows that it suffices to assume that the smallest (most negative) eigenvalue of $G$ is at least $-\lambda$ to conclude that the average degree is at least $\alpha d-\lambda(1-\alpha)$. (The assumption that the second largest eigenvalue is at most $\lambda$ provides an upper bound of $\alpha d+\lambda(1-\alpha)$ for this average degree).

For random Cayley graphs of $\mathbb{Z}_{2}^{n}$ with at least some $C n$ generators, $\lambda$ is smaller than, say, $d / 2$ with high probability, as proved in [3].

Proposition $5.2([3])$. For every $1>\delta>0$ there exists a $C(\delta)>0$ such that the following holds. Let $B$ be a group of order $n$, let $S$ be a set of $C(\delta) \log _{2}(n)$ random elements of $B$, where $S=S^{-1}$ and let $G$ be the Cayley graph of $B$ with respect to $S$. Then, with high probability (that is, with probability that tends to 1 as $n$ tends to infinity) the absolute value of every eigenvalue of $G$ but the top one is at most $(1-\delta)|S|$.

Taking $\delta=1 / 2$ this shows that the lower bound in Theorem 1.1 can be improved to $|S| / 4$ for most Cayley graphs of $\mathbb{Z}_{2}^{n}$ with at least $C(1 / 4) n$ generators.

Theorem 1.2 shows that any connected Cayley graph of $\mathbb{Z}_{2}^{n}$ with at most $n+1$ generators admits a unitary signing. It is not difficult to give additional examples of Cayley graphs of this group with such a signing. In particular the tensor product of any two graphs with a unitary signing admits a unitary signing, as follows by the mixed product property. Any $m$ by $m$ Hadamard matrix provides an orthogonal (and hence unitary) signing of the complete graph with loops on $m$ vertices in each vertex class. Tensor products of such a graph and graphs covered by Theorem 1.2 are graphs admitting unitary signings. On the other hand, there are lots of Cayley graphs of $\mathbb{Z}_{2}^{n}$ that do not admit such a signing. A large family of examples are ones in which the generating set $S$ is a Sidon set with more than $2 n+1$ elements. To prove 


\section{Unitary Signings and InduCED Subgraphs of CAYley Graphs of $\mathbb{Z}_{2}^{n}$}

this note that if $S$ is a Sidon set, a unitary signing (squaring to $|S| I)$ must be the sum of $|S|$ anticommuting edge signings. This is because if the unitary signing is decomposed into edge signings, $e_{1}+\cdots+e_{|S|}$, in the square of this sum the nonzero entries of any product of two matrices $e_{i}, e_{j}$ can intersect those of $e_{p}, e_{q}$ only if $\{i, j\}=\{p, q\}$. Thus the only way for the sum to square to $|S| I$ is for all of the edge signings to anticommute with each other.

It is known (c.f. [8]) that the maximum possible number of complex invertible anticommuting $N$ by $N$ matrices is $2 \log _{2} N+1$. In our case $N=2^{n}$ and thus we cannot have more than $2 n+1$ anticommuting matrices corresponding to edge signing (note that each such matrix is invertible, as its square is the identity). There are, however, much larger Sidon sets in $\mathbb{Z}_{2}^{n}$, of size $2^{n / 2}$ (given by the columns of the parity check matrix of a BCH code with designed distance 5). Any Cayley graph of $\mathbb{Z}_{2}^{n}$ with a generating set which is a subset of more than $2 n+1$ elements of such a large Sidon set cannot have a unitary signing.

It will be interesting to understand better which (Cayley or non-Cayley) graphs admit unitary signings. It also seems interesting to investigate the possible analogs of Theorem 1.1 for Cayley graphs of other groups.

\section{Acknowledgments}

We thank two anonymous reviewers for helpful suggestions and comments.

\section{References}

[1] N. Alon and F. Chung, Explicit construction of linear sized tolerant networks, Discrete Math. 72 (1988), 15-19. 10

[2] N. Alon and V. D. Milman, $\lambda_{1}$, isoperimetric inequalities for graphs, and superconcentrators, $J$. Combin. Theory, Ser. B 38 (1985), 73-88. 9

[3] N. Alon and Y. Roichman, Random Cayley graphs and expanders, Random Struct Algorithms 5 (1994), 271-284. 10

[4] F. Belardo, S. M. Cioabă, J. H. Koolen and J. Wang, Open problems in the spectral theory of signed graphs, The Art of Discrete and Applied Mathematics 1 (2018), P2.10 2, 8, 9

[5] A.E. Brouwer, A.M. Cohen and A. Neumaier, Distance-Regular Graphs, Springer, 1989. 6

[6] F. Chung, Z. Füredi, R. Graham and P. Seymour, On induced subgraphs of the cube, J. Combin. Theory, Ser. A 49 (1988), 180-187. 2, 10

[7] C. Gotsman and N. Linial, The equivalence of two problems on the cube, J. Combin. Theory, Ser. A 61 (1992), 142-146. 1

[8] P. Hrubeš, On families of anticommuting matrices, Linear Algebra Appl. 493 (2016), 494-507. 11

[9] H. Huang, Induced subgraphs of hypercubes and a proof of the sensitivity conjecture, Ann. Math. 190 (2019), 949-955. 1, 3, 4 
[10] S. G. Hwang, Cauchy's interlace theorem for eigenvalues of hermitian matrices, Am. Math. Mon. 111 (2004), 157-159. 2

[11] L. Lovász, Combinatorial Problems and Exercises, North-Holland Publishing Co., Amsterdam-New York, 1979. 9

[12] J. McKee and C. Smyth, Integer symmetric matrices having all their eigenvalues in the interval [-2,2], J. Algebra 317 (2007), 260-290. 9

[13] L. Mancinska, I. Pivotto, D. E. Roberson and G. F. Royle, Cores of cubelike graphs, European J. Combin. 87 (2020), 103092, 16 pp. 2

[14] N. Nisan, and M. Szegedy, On the degree of boolean functions as real polynomials, Computational Complexity 4 (1994), 301-313. 1

\title{
AUTHORS
}

\author{
Noga Alon \\ Department of Mathematics, \\ Princeton University, \\ Princeton, USA \\ and
}

Schools of Mathematics and Computer Science,

Tel Aviv University,

Tel Aviv, Israel.

nogaa@tau.ac.il

Kai Zheng

Department of Mathematics,

Princeton University,

Princeton, USA.

kzheng@princeton.edu 